\title{
A Comparative Analysis of Techniques for PAPR Reduction of OFDM Signals
}

\author{
Miloš Janjić and Nataša Nešković
}

\begin{abstract}
In this paper the problem of high Peak-toAverage Power Ratio (PAPR) in Orthogonal FrequencyDivision Multiplexing (OFDM) signals is studied. Besides describing three techniques for PAPR reduction, SeLective Mapping (SLM), Partial Transmit Sequence (PTS) and Interleaving, a detailed analysis of the performances of these techniques for various values of relevant parameters (number of phase sequences, number of interleavers, number of phase factors, number of subblocks) depending on applied technique, is carried out. Simulation of these techniques is run in Matlab software. Results are presented in the form of Complementary Cumulative Distribution Function (CCDF) curves for PAPR of 30000 randomly generated OFDM symbols. Simulations are performed for OFDM signals with 32 and 256 subcarriers, oversampled by a factor of 4 . A detailed comparison of these techniques is made based on Matlab simulation results.
\end{abstract}

Keywords - Interleaving, OFDM, PAPR, PTS, SLM.

\section{INTRODUCTION}

$\mathrm{O}$ RTHOGONAL Frequency-Division Multiplexing (OFDM) is a very attractive transmission technique, which is employed in many present communication systems, and it is a strong candidate for use in future high data rate wireless multimedia systems. OFDM has several favorable properties like high spectral efficiency, robustness to multipath fading, simple equalization in frequency domain, simple implementation using Inverse Fast Fourier Transform (IFFT). On the other hand, the major drawbacks are frequency offset sensitivity, high Peak-to-Average Power Ratio (PAPR), and phase noise sensitivity.

A high PAPR of OFDM signals is the result of the nature of IFFT, where each output sample is the sum of a large number of sinusoids. In most cases these sinusoids are added destructively, leading to a relatively low mean signal power. However, on very rare occasions all the sinusoids, or more often a large number of them, have the same phase so they add constructively, which produces very high signal peaks. Most radio systems employ a High Power Amplifier (HPA) in the transmitter to obtain

Paper received March 17, 2014; accepted June 10, 2014. Date of publication July 31,2014 . The associate editor coordinating the review of this manuscript and approving it for publication was Prof. Zorica Nikolić.

This paper is a revised and expanded version of the paper presented at the 21th Telecommunications Forum TELFOR 2013.

Miloš Janjić, Student of PhD study, University of Belgrade - School of Electrical Engineering, milos_janjic@yahoo.com.

Nataša Nešković, University of Belgrade - School of Electrical Engineering, natasha@etf.rs. sufficient transmit power. The HPA is usually the largest power consumer in the transmitter, so it should work at maximum efficiency. Here, the term efficiency is a measure of amplifier's ability to convert the DC power of the supply into the RF signal power at the output. Efficiency is particularly important in the uplink because it allows a longer life of mobile terminal battery, smaller devices, etc. Therefore, it is optimal to operate the HPA at or near the saturation region. This is feasible for signals with a constant amplitude. However, if the HPA is operated near the saturation in the case of signals with a high PAPR, it will work in its non-linear region during high peak intervals. Since the OFDM signal is composed of a large number of closely spaced subcarriers, a great number of intermodulation products will appear at the HPA output. Some intermodulation products will be inside the OFDM signal bandwidth, which will cause loss of orthogonality between subcarriers, and will lead to BER degradation. Intermodulaton products outside the OFDM signal bandwidth will make interference to signals in adjacent parts of the spectrum. In order to prevent operation in a non-linear region, it is required that HPA work with large power back-off values, i.e., much lower values than those that lead the HPA to saturation. Hence, large variations in the instantaneous power of transmitted signal cause reduced HPA efficiency. Moreover, HPAs whose linear region spans a wide dynamic range are very expensive. Signals with a high PAPR require A/D and D/A converters with a wide dynamic range and a large number of quantization levels to limit the quantization noise. These converters are very costly, too. Consequently, we should try to reduce the OFDM signal PAPR before it is passed to the D/A converter and HPA. For this purpose a number of techniques have been proposed, three of which are described and comparatively analyzed in this paper.

The second section gives the theoretical basics regarding the term PAPR. The third section contains a brief description of three techniques for PAPR reduction, SLM (SeLective Mapping), PTS (Partial Transmit Sequence), and Interleaving. In the fourth section Matlab simulation results are presented, based on which a comparative analysis of the three techniques is carried out. The paper is concluded in the fifth section.

\section{PAPR OF OFDM}

The PAPR of OFDM signals is defined as the ratio between the maximum instantaneous signal power and its average power over one symbol length. In OFDM signal transmission, in baseband we deal with discrete signal 
samples, but there is a need to estimate the PAPR of the continuous signal after the $\mathrm{D} / \mathrm{A}$ conversion. In order to evaluate the PAPR of the continuous signal, it is not sufficient to observe the signal samples at the output of IFFT, because this discrete signal won't contain all the peaks that will appear in the continuous signal. Therefore, it is necessary to oversample the discrete signal. It is stated in [1] that oversampling by factor $L=4$ is sufficient to get accurate PAPR estimation of the continuous signal. The PAPR of the RF OFDM signal is twice the PAPR of the baseband signal [1]. In the analysis of PAPR reduction techniques the baseband OFDM signal is usually observed.

Since the OFDM subcarriers are orthogonal, the average power of OFDM signals is equal to the sum of the average powers of subcarriers. The maximum power occurs when all of the $N$ subcarrier components happen to be added with identical phases, so that their amplitudes are added together. In PSK/OFDM systems, where all subcarrier signals have equal amplitude, the maximum instantaneous power of the OFDM signal is $N$ times its average power. More PAPR is expected for QAM, because the amplitudes of QAM symbols are not equal for all constellation points. The probability of the occurrence of the maximum power signal is very low and it decreases as $N$ increases. Therefore we are interested in finding the probability that the signal power is out of the linear range of the HPA. Towards this end, we first consider the amplitude distribution of the OFDM signal at the output of IFFT. While the input signals of $N$-point IFFT have the independent and finite magnitudes which are uniformly distributed for QPSK and QAM, we can assume that the real and imaginary parts of the time-domain complex OFDM signal have an asymptotically Gaussian distribution for a sufficiently large number of subcarriers by the central limit theorem [2]. Then the amplitude of the time-domain complex OFDM signal follows a Rayleigh distribution.

\section{TECHNIQUES FOR PAPR REDUCTION}

A number of techniques have been proposed for PAPR reduction. They can be divided into two main groups: simple techniques that cause distortions in the signal, and more complex techniques that don't distort the signal.

Among PAPR reduction techniques that don't cause distortions, very popular are so-called probabilistic techniques. Three techniques from this group, SLM, PTS, and Interleaving are analyzed in this paper. The basic idea in these techniques is to generate a set of alternative signals from the original signal block at the input to IFFT, all of them containing the same information, and to transmit one of them with the minimum PAPR value. These techniques reduce the probability of incurring high signal peaks, and cannot guarantee the PAPR below a specified level. An increase in transmit power is not required. This approach is applicable to all types of modulation and any number of subcarriers. On the other hand, in order for the receiver to be able to recover the original data block, the information about the selected alternative signal for transmission should be sent as side information. It is essential that this information be correctly received, otherwise the whole OFDM symbol will be corrupted.

\section{A. SLM technique}

Prior to being fed to IFFT input, data block $\mathbf{X}=[X[0], X[1], \ldots, X[\mathrm{~N}-1]]^{T}$ is multiplied with $U$ different phase sequences (vectors), $P^{u}=\left[P_{0}^{u}, P_{1}^{u}, \ldots, P_{N-1}^{u}\right]^{T}$, where $P_{v}^{u}=e^{j \varphi_{v}^{u}}$, and $\varphi_{v}^{u} \in$ $[0,2 \pi)$, for $v=0,1, \ldots, N-1$, and $u=1,2, \ldots U$, which produces a modified data block $\mathbf{X}^{u}=\left[X^{u}[0], X^{u}[1], \ldots, X^{u}[N-1]\right]^{T} \quad$ [2]. IFFT of $U$ independent sequences of length $N$ is taken to produce the sequences $\quad \mathbf{x}^{u}=\left[x^{u}[0], x^{u}[1], \ldots, x^{u}[N-1]\right]^{T}$, among which the one $\tilde{\mathbf{x}}=\mathbf{x}^{\widetilde{u}}$, with the lowest PAPR is selected for transmission. $P^{1}$ is the all-one vector of length $N$, to include the unmodified original sequence in the set of modified sequences. In order to reduce the complexity, the set of allowed elements of phase sequences, $\varphi_{v}^{u}$, is usually limited to several values. Information about the selected phase sequence is transmitted to the receiver as side information. At the receiver, the reverse operation is performed to recover the original data block. For implementation of the SLM technique $U$ IFFT blocks and $\log _{2} U$ bits for side information are required. The amount of PAPR reduction depends on the number and design of phase sequences [3].

\section{B. PTS technique}

PTS technique partitions an input data block of length $N$ into $V$ non-overlapping subblocks of equal size, $\mathbf{X}=$ $\left[\mathbf{X}^{1}, \mathbf{X}^{2}, \ldots, \mathbf{X}^{\mathrm{V}}\right]^{T}$, where $\mathbf{X}^{i}$ are the subblocks that are consecutively located. Unlike the SLM technique in which scrambling is applied to all subcarriers, scrambling (rotating its phase independently) is applied to each subblock in the PTS technique [2]. Then each partitioned subblock is multiplied by a corresponding phase factor $b^{v}=e^{j \varphi^{v}}, \quad v=1,2, \ldots, V, \quad$ and brought to $N / V$ corresponding inputs to a different IFFT block of length $N$, while the rest of the inputs are filled with zeros, so that the output signal can be expressed as follows

$$
\begin{gathered}
\mathbf{x}=\operatorname{IFFT}\left\{\sum_{v=1}^{V} b^{v} \mathbf{X}^{v}\right\} \\
=\sum_{v=1}^{V} b^{v} \cdot \operatorname{IFFTT}\left\{\mathbf{X}^{v}\right\} \\
=\sum_{v=1}^{V} b^{v} \mathbf{x}^{v} .
\end{gathered}
$$

The phase vector is chosen so that the PAPR is minimized. Then, the corresponding time-domain signal with the lowest PAPR can be represented as [2]

$$
\tilde{\mathbf{x}}=\sum_{v=1}^{V} \tilde{b}^{v} \mathbf{x}^{v} \text {. }
$$

In order to reduce the search complexity, the set of potential values of phase factors $\left\{b^{v}\right\}_{v=1}^{V}$ is usually limited. As the set of allowed phase factors is $\mathbf{b}=$ $\left\{e^{j 2 \pi i / W}, i=0,1, \ldots, W-1\right\}$, and if we assume that $b^{1}=1, W^{V-1}$ phase vectors should be searched to find the optimum set of phase factors. Therefore, the search complexity increases exponentially with the number of subblocks. The implementation of the PTS technique requires $V$ IFFT operations for every data block, and $\log _{2} W^{V}$ bits for side information, or $\log _{2} W^{V-1}$, if we assume that $b^{1}=1$. 


\section{Interleaving technique}

In [5] Interleaving technique for PAPR reduction of OFDM signals has been proposed. The technique employs $K-1$ interleavers, placed in front of IFFT blocks, each one at its output producing a different permuted sequence from the same information sequence comprised of $N$ symbols. From $K$ signals bearing the same information (the original sequence and K-1 permuted sequences) the one with the minimum PAPR at the output of IFFT is selected for transmission. $\log _{2} K$ bits are required for side information. Two interleaver types, random and periodic, are used. A random interleaver, which is implemented in the simulation, is described here.

A random interleaver is a block interleaver that operates onto a block of $N$ symbols and reorders or permutes them in a pseudo random order; i.e. symbol sequence $\mathbf{X}=$ $\left(X_{0}, X_{1}, \ldots, X_{n}, \ldots, X_{N-1}\right) \quad$ becomes $\quad\left\{\mathbf{X}^{n}\right\} \quad=$ $\left(X_{\pi(0)}, X_{\pi(1)}, \ldots, X_{\pi(n)}, \ldots, X_{\pi(N-1)}\right)$, where $\{n\} \leftrightarrow\{\pi(n)\}$ is a one to one mapping and $\pi(n) \in\{0,1, \ldots, N-1\}$, for all $n[5] . \mathbf{X}^{0}$ is the original sequence, i.e. $\mathbf{X}^{0}=\mathbf{X}$. Both the transmitter and the receiver store the permutation indices, $\{\pi(n)\}$, in memory, which makes interleaving and deinterleaving very simple operations. The amount of PAPR reduction depends on the number and design of interleavers.

\section{Simulation Results}

Simulations were run in Matlab software. Empirical CCDF (Complementary Cumulative Distribution Function) was calculated for PAPR of 30000 randomly generated OFDM symbols. Simulations for OFDM signals with 32 and 256 subcarriers were performed, in order to investigate the dependence of CCDF for OFDM signal PAPR and reduction obtained by employment of various techniques on the number of subcarriers. The modulation used on the subcarriers was QPSK. CCDF was calculated using OFDM signal samples in baseband oversampled by a factor of 4. Figs. 1 and 2 show the influence of oversampling on CCDF curves for the OFDM signals with 32 and 256 subcarriers, respectively. Based on the figures, we find it appropriate to have the signal at the output of IFFT oversampled by factor $L=4$. Obtained PAPR is roughly $0.2-0.5 \mathrm{~dB}$ higher compared to the nonoversampled signal. On the other hand, oversampling by larger factors would result in a very large number of signal samples, without a significant benefit concerning estimation of the continuous signal PAPR.

Obtained CCDF curves for OFDM signals with 32 and 256 subcarriers after application of the three discussed techniques are shown in Figs. 3 and 4, respectively. By observation of the curves for the original signal we notice that PAPR increases as the number of subcarriers increases. In the following part of the paper results obtained by use of the SLM, PTS, and Interleaving techniques are analyzed and compared.

\section{A. SLM technique}

The set of allowed phase factors was comprised of 4 elements, $\{1,-1, j,-j\}$. Looking at either of Figs. 3 and 4 we observe that PAPR is reduced with the increase in the number of phase sequences, $U$, resulting from an increased number of options. However, if $U$ is increased, the number of required IFFT blocks increases, as well as, the number of bits for side information. Benefit from increasing $U$ decreases as $U$ increases, as it can be seen that curves in Figs. 3 and 4 are closer to each other, although the relative increase of $U$ is constant (2 times). PAPR reduction using the SLM technique is greater for OFDM signals with fewer subcarriers. These claims are supported by the following observations from Figs. 3 and 4. Consider the SLM technique with $U=4$. At a probability of $0.1, \mathrm{CCDF}$ value for the OFDM signal with 32 subcarriers is reduced by $1.5 \mathrm{~dB}$ compared to the original signal, whereas for the signal with 256 subcarriers the reduction amounts to 1.3 $\mathrm{dB}$. Hence, the difference is $0.2 \mathrm{~dB}$ in favor of the signal with fewer subcarriers. The difference is more significant at lower probabilities. At a probability of $0.0001, \mathrm{CCDF}$ value for the signal with 32 subcarriers is reduced by $3 \mathrm{~dB}$, while for the signal with 256 subcarriers PAPR reduction equals $2.5 \mathrm{~dB}$, i.e., the difference is $0.5 \mathrm{~dB}$. Now, let us consider the SLM technique with $U=32$. At a probability of 0.1, CCDF value for the OFDM signal with 32 subcarriers is reduced by $2.8 \mathrm{~dB}$ compared to the original signal, whereas for the signal with 256 subcarriers the

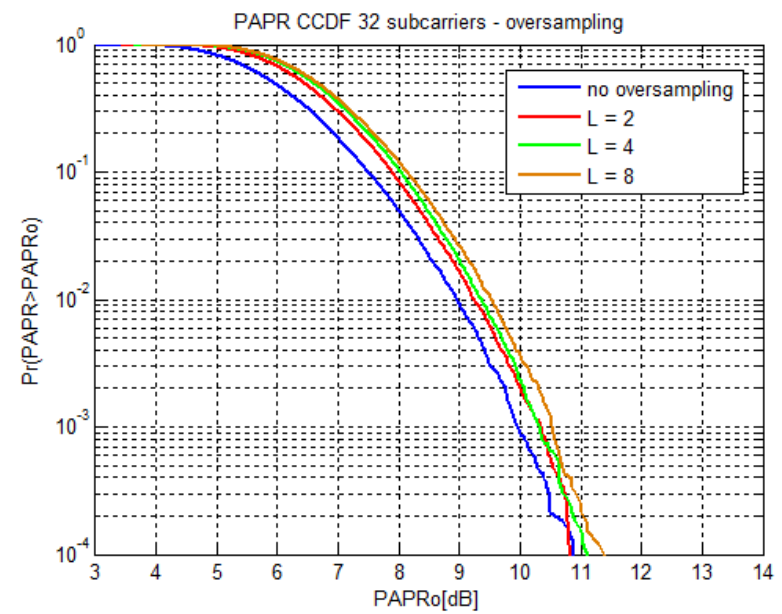

Fig. 1. Dependence of CCDF for PAPR of OFDM signals with 32 subcarriers on oversampling factor.

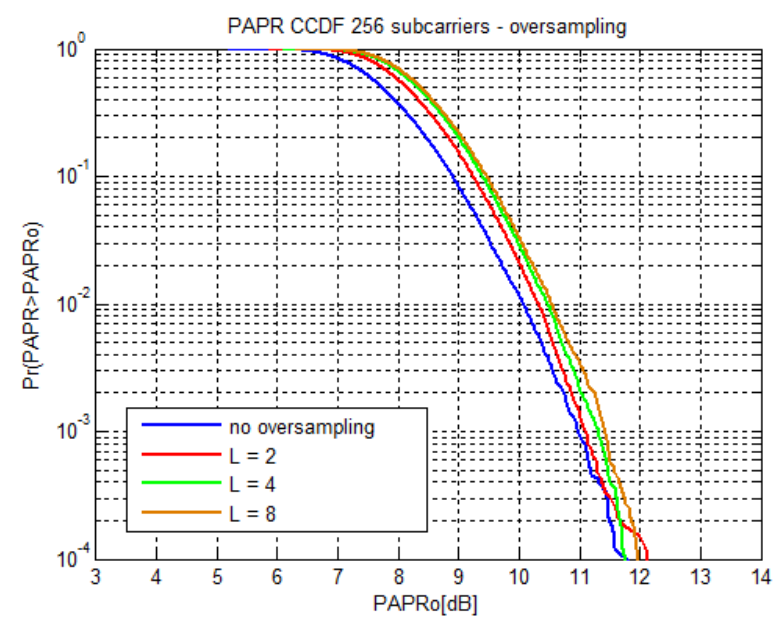

Fig. 2. Dependence of CCDF for PAPR of OFDM signals with 256 subcarriers on oversampling factor. 


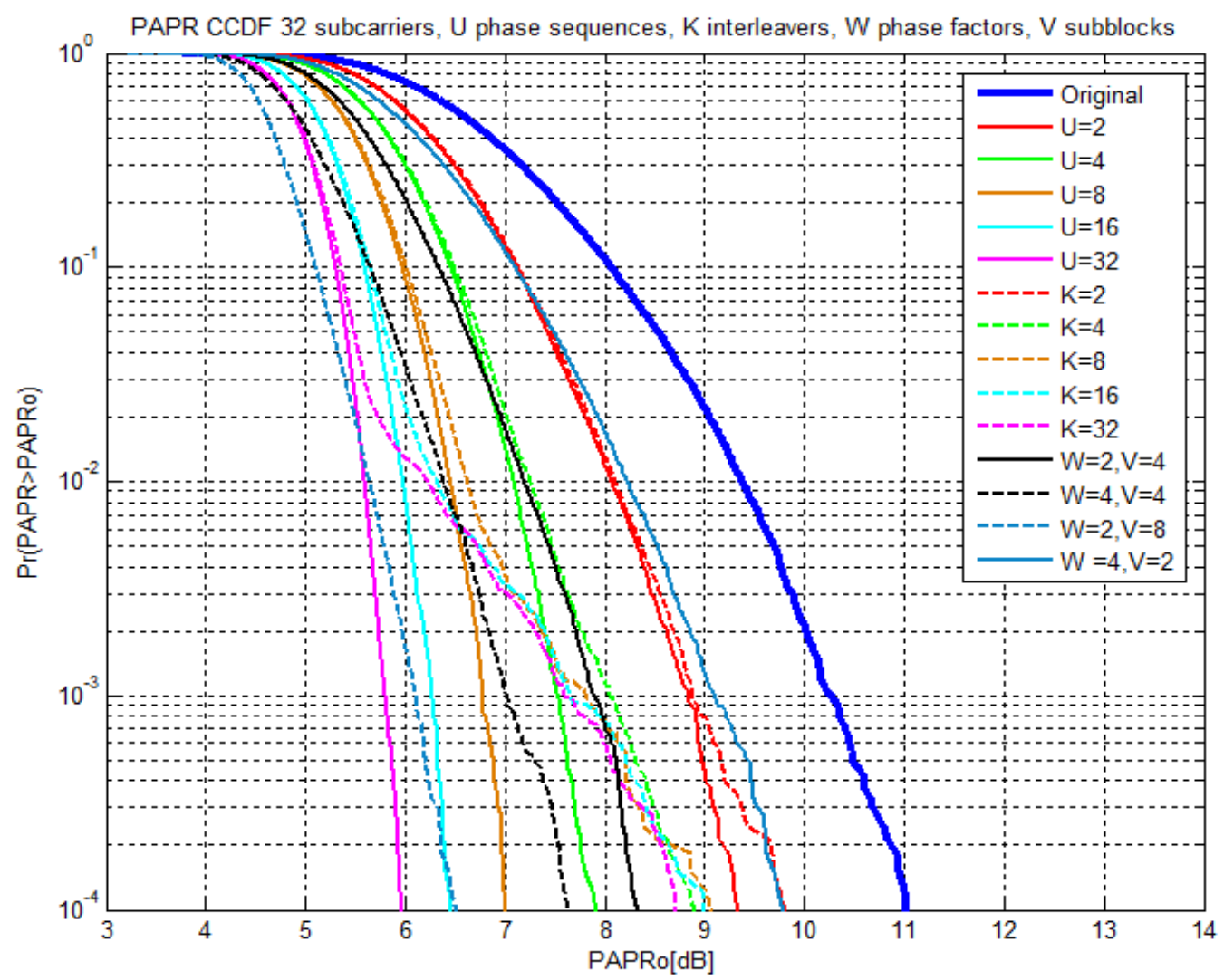

Fig. 3. CCDFs for PAPR of OFDM signals with 32 subcarriers.

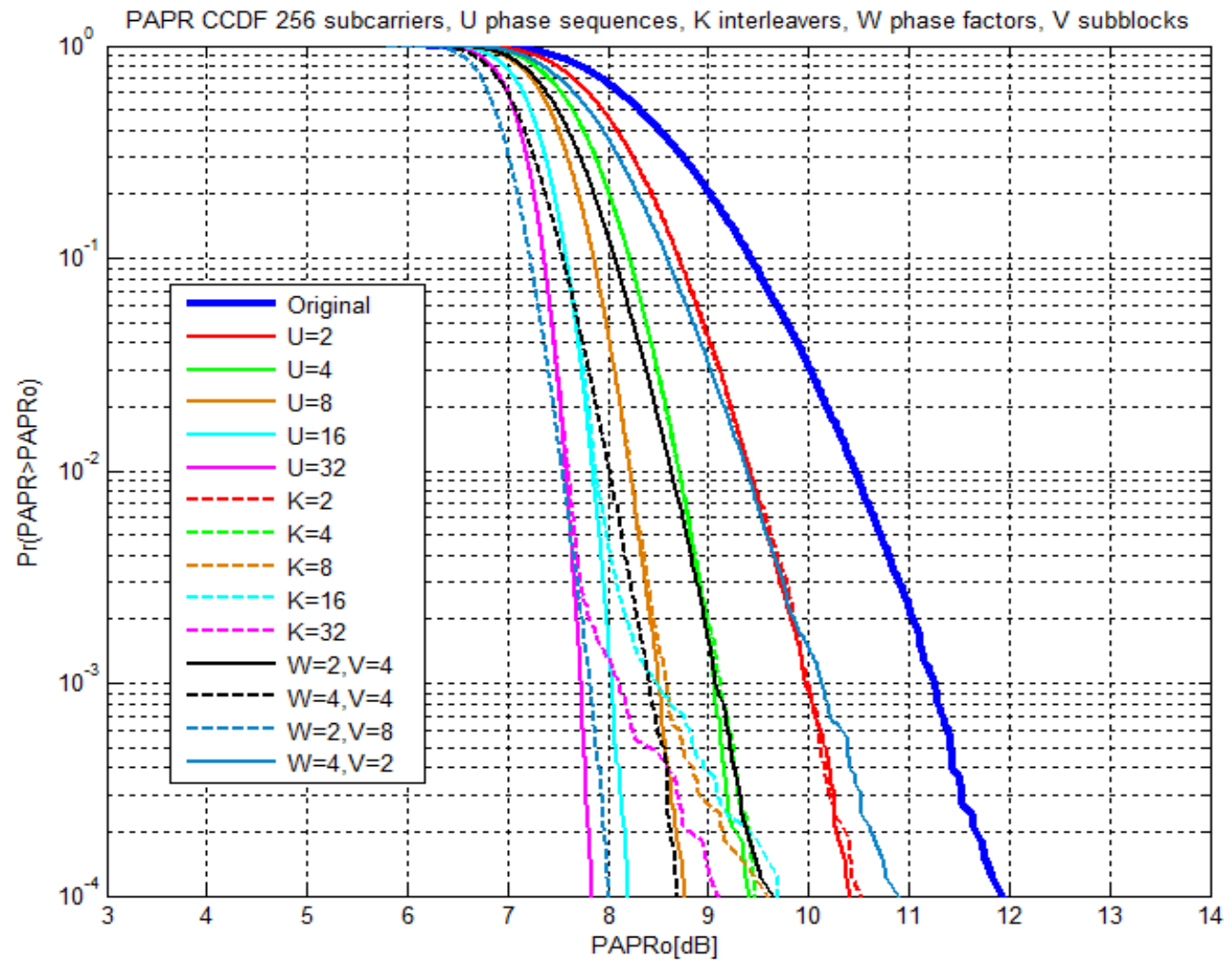

Fig. 4. CCDFs for PAPR of OFDM signals with 256 subcarriers. 
reduction is equal to $2.1 \mathrm{~dB}$. Hence, the difference is $0.7 \mathrm{~dB}$. At lower probabilities the difference is even larger. At probability of $0.0001, \mathrm{CCDF}$ value for the signal with 32 subcarriers is reduced by $5 \mathrm{~dB}$, whereas for the signal with 256 subcarriers PAPR reduction equals $4.1 \mathrm{~dB}$, i.e. the difference is $0.9 \mathrm{~dB}$. Regarding CCDF for the SLM technique with a particular number of phase sequences, PAPR reduction compared to the original signal increases as values of PAPR increase, in other words, as CCDF probabilities decrease.

\section{B. Interleaving technique}

For the same reason as in the case of the SLM technique, PAPR is reduced with the increase in the number of interleavers. However, if $K$ is increased, the number of required IFFT blocks increases, as well as the number of bits for side information. Improvement is reduced as $K$ increases, as in the case of the SLM technique. Consider the Interleaving technique with $K=4$. At a probability of 0.1, CCDF value for the OFDM signal with 32 subcarriers is reduced by $1.5 \mathrm{~dB}$ compared to the original signal, whereas for the signal employing 256 subcarriers the reduction amounts to $1.3 \mathrm{~dB}$. Therefore, the difference is $0.2 \mathrm{~dB}$ in favor of the OFDM signal with a smaller number of subcarriers. At lower probabilities, we obtained different results. At a probability of 0.0001 , CCDF value for the signal with 32 subcarriers is reduced by $2 \mathrm{~dB}$, while for the OFDM signal with 256 subcarriers PAPR reduction equals $2.4 \mathrm{~dB}$, i.e. the difference is $0.4 \mathrm{~dB}$ in favor of the signal employing a greater number of subcarriers. Now, let us consider the Interleaving technique with $K=32$. At a probability of $0.1, \mathrm{CCDF}$ value for the OFDM signal with 32 subcarriers is reduced by $2.7 \mathrm{~dB}$ compared to the original signal, whereas for the signal employing 256 subcarriers the reduction equals $2.1 \mathrm{~dB}$, i.e., the difference is $0.7 \mathrm{~dB}$ in favor of the OFDM signal with a smaller number of subcarriers. Again, results are different at lower probabilities. At a probability of 0.0001, CCDF value for the signal with 32 subcarriers is reduced by $2.3 \mathrm{~dB}$ compared to the original signal, while for the signal with 256 subcarriers PAPR reduction amounts to $2.8 \mathrm{~dB}$, i.e. the difference is $0.5 \mathrm{~dB}$ in favor of the signal employing a larger number of subcarriers.

Regarding CCDF for the Interleaving technique with a particular number of interleavers, starting from higher probabilities, the reduction of PAPR increases as probability decreases, but after a certain probability, the reduction is approximately constant and doesn't depend on probability. This probability value decreases with the number of subcarriers and increases with the number of interleavers.

\section{SLM and Interleaving techniques comparison}

It is clear from Figs. 3 and 4 that curves for the SLM and the Interleaving are almost identical at higher probabilities, but starting from the above mentioned probability value, there is a difference between performances of the two techniques in favor of the SLM technique, which becomes more significant as probability decreases. This statement is supported by the following observations from Figs. 3 and 4. Let us consider two cases, with 4 and 32 alternative signals, respectively. At a probability of 0.1 , for the signals with 32 subcarriers the curves can be considered equal. At a probability of 0.0001 , the difference in PAPR reduction amounts to $1 \mathrm{~dB}$ in the case of 4 alternative signals, and $2.7 \mathrm{~dB}$ for 32 alternative signals. Regarding the 256-subcarrier OFDM signal, the curves are identical at a probability of 0.1 . At a probability of 0.0001 , the difference is only $0.1 \mathrm{~dB}$ in the case of 4 alternative signals, and $1.3 \mathrm{~dB}$ for 32 alternative signals. The SLM technique uses multiplication and IFFT blocks, and it is more complex for implementation than the Interleaving technique, that uses interleavers and IFFT blocks. The techniques require the same number of bits for side information in order to generate a certain number of alternative signals. The SLM technique offers better results at low probabilities, in other words, the SLM outperforms the Interleaving in terms of the reduction of extremely high signal peaks. A positive feature of the Interleaving technique is that it shows the best performances at probabilities higher than 0.01 , so it is in most cases on a par with the SLM.

\section{PTS technique}

From Figs. 3 and 4 it is easily noticed that by increasing either the number of subblocks, $V$, or the number of phase factors, $W$, PAPR reduction increases, which is expected since the number of alternative signals increases. Better results are obtained by increasing $V$ than by increasing $W$, but, on the other hand, in addition to a more significant increase in the number of bits for side information, the number of IFFT blocks increases, as well. PAPR reduction by the PTS technique is greater for OFDM signals using a smaller number of subcarriers. Consider the PTS technique with $W=2$ and $V=8$. At a probability of 0.1 , CCDF value for the OFDM signal employing 32 subcarriers is reduced by $3 \mathrm{~dB}$ compared to the original signal, whereas for the signal with 256 subcarriers the reduction is equal to $2.3 \mathrm{~dB}$. The difference is $0.7 \mathrm{~dB}$ in favor of the signal using 32 subcarriers. At a probability of $0.0001, \mathrm{CCDF}$ value for the signal with 32 subcarriers is reduced by $4.6 \mathrm{~dB}$ compared to the original signal, while in the case of a 256-subcarrier OFDM signal PAPR is reduced by $3.9 \mathrm{~dB}$, i.e. the difference equals $0.5 \mathrm{~dB}$. Regarding CCDF for the PTS technique with certain values of $V$ and $W$, PAPR reduction compared to the original signal increases as values of PAPR increase, in other words, as CCDF probabilities decrease.

It can be seen from Figs. 3 and 4 that for the same number of alternative signals, e.g., $16(W=2, V=4$, and $W=4, V=2)$ or $256(W=4, V=4$, and $W=2, V=8)$, better results are obtained in the cases with a greater number of subblocks. On the other hand, a larger number of IFFT blocks are required for implementation in these cases. For the OFDM signal with 32 subcarriers, in the case of 16 alternative signals, the difference is $0.8 \mathrm{~dB}$ at a probability of 0.1 , and $1.6 \mathrm{~dB}$ at a probability of 0.0001 , while in the case of 256 alternative signals, it is equal to $0.6 \mathrm{~dB}$ at a probability of 0.1 , and $1.1 \mathrm{~dB}$ at a probability of 0.0001 . For the signal employing 256 subcarriers, in the case of 16 alternative signals, the difference equals $0.4 \mathrm{~dB}$ 
at a probability of 0.1 , and $1.2 \mathrm{~dB}$ at a probability of 0.0001 , while in the case of 256 alternative signals, it is $0.3 \mathrm{~dB}$ at a probability of 0.1 , and $0.7 \mathrm{~dB}$ at a probability of 0.0001. Therefore, the difference increases as probability values decrease, and it decreases with the increase in the number of subcarriers, as well as the number of alternative signals.

\section{E. SLM and PTS techniques comparison}

If we consider a case when the techniques employ the same number of IFFT blocks, e.g. 4, and the same number of phase factors, 4 , it is clear that the PTS technique offers a better performance, because it generates a larger number of alternative signals, 256 (PTS) versus 4 (SLM). However, the PTS requires 8 bits for side information, whereas the SLM needs only 2 . In the case of 32 subcarriers, the difference in PAPR reduction is $0.8 \mathrm{~dB}$ at a probability of 0.1 , and $0.4 \mathrm{~dB}$ at a probability of 0.0001 . For 256 subcarriers, the difference equals $0.6 \mathrm{~dB}$ and it is approximately constant over all probabilities.

Now, let us discuss a situation when the SLM and the PTS produce the same number of alternative signals, e.g. $U=16$ and $V=4, W=2$ (better of the two discussed options when the PTS generates 16 alternative signals). From Figs. 3 and 4 it is obvious that the SLM outperforms the PTS. The reason for this is the fact that the SLM can produce multiple time domain OFDM signals that are asymptotically independent, whereas the alternative signals generated by the PTS are interdependent [1]. The difference in the PAPR reduction is slightly larger for the 32-subcarrier signal, in which case it equals $0.7 \mathrm{~dB}$ at a probability of 0.1 , and $1.7 \mathrm{~dB}$ at a probability of 0.0001 . In the case of a 256-subcarrier signal the difference is 0.6 $\mathrm{dB}$ at a probability of 0.1 , and $1.5 \mathrm{~dB}$ at a probability of 0.0001 . Therefore, in both cases the difference increases as probability decreases. However, in this situation the SLM employs 16 IFFT blocks, while the PTS uses only 4.

\section{CONCLUSION}

On the basis of the presented simulation results, it can be concluded that the PTS technique has the greatest potential for PAPR reduction. It offers the best performance for a certain number of IFFT blocks because it generates the largest number of alternative signals. On the other hand, a very large number of alternative signals cause a large number of required bits for side information and higher search complexity, as well. The SLM technique offers the number of alternative signals equal to the number of employed IFFT blocks. However, for the same number of alternative signals, the SLM technique shows the best performance, but in this case its implementation is the most complex. The Interleaving is the simplest technique for implementation, in the range of higher probabilities it has similar performances to the SLM technique, but at lower probabilities, i.e. in the range of extremely high peaks, it performs significantly poorer compared to the SLM.

\section{REFERENCES}

[1] T. Jiang and Y. Wu, "An overview: Peak-to-average power ratio reduction techniques for OFDM signals", IEEE Transactions on Broadcasting, Vol. 54, No. 2, pp. 257-268, June 2008.

[2] Y. S. Cho, J. Kim, W. Y. Yang, C. G. Kang, "MIMO-OFDM Wireless Communications with MATLAB", John Wiley and Sons (Asia) Pte Limited, Singapore, 2010.

[3] S. H. Han and J. H. Lee, "An overview of peak-to-average power ratio reduction techniques for multicarrier transmission", IEEE Wireless Communications, pp. 56-65, April 2005.

[4] V. Vijayarangan and R. Sukanesh, "An overview of techniques for reducing peak to average power ratio and its selection criteria for orthogonal frequency division multiplexing radio systems", Journal of Theoretical and Applied Information Technology, 2009.

[5] A. D. S. Jayalath and C. Tellambura, "The use of interleaving to reduce peak to average power ratio of an OFDM signal", IEEE Global Telecommunications Conference, pp. 82-86, San Francisco, CA, 2000. 